\title{
Statistical Physics of DNA melting: Unveiling the artificial corrections for self-complementary sequences
}

\author{
${ }_{5}^{5}$ C. A. Plata ${ }^{1,}$, S. Marni ${ }^{2}$, A. Maritan ${ }^{1}$, T. Bellini ${ }^{2}$, and S. Suweis ${ }^{1, \dagger}$
}

\begin{abstract}
${ }_{11}$ ABSTRACT DNA hybridization is at the heart of countless biological and biotechnological processes. Its theoretical modeling 12 played a crucial role, since it has enabled extracting the relevant thermodynamic parameters from systematic measurements of ${ }_{13}$ DNA melting curves. However, in its current state, hybridization modelling requires introducing an extra entropic contribution 14 in self-complementary sequences that lacks any biophysical meaning. In this article, we propose a framework based on 15 statistical physics to describe DNA hybridization and melting in an arbitrary mixture of DNA strands. In particular, we are able to ${ }_{16}$ analytically derive closed expressions of the system partition functions for any number $N$ of strings, and explicitly calculate them 17 in two paradigmatic situations: (i) a system made of self-complementary sequences and (ii) a system comprising two mutually ${ }_{18}$ complementary sequences. We derive the melting curve in the thermodynamic limit $(N \rightarrow \infty)$ of our description, which differs 19 from the expression commonly used to evaluate the melting of self-complementary systems in that it does not require correcting ${ }_{20}$ terms. We provide a thorough study comprising limit cases and alternative approaches showing how our framework can give a ${ }_{21}$ comprehensive view of hybridization and melting phenomena.
\end{abstract}

SIGNIFICANCE In this study, we provide a transparent derivation of the melting curves of DNA duplexes using basic tools of statistical mechanics. We find that in the case of self-complementary sequences, our expression differs from the one used in literature, which is generally amended by the introduction of a phenomenological correction which in our approach becomes unnecessary. By offering a clean formal description of DNA hybridization, our approach sharpens our understanding of DNA interactions and opens the way to study the pairing of DNA oligomers away from any thermodynamic limit.

\section{${ }_{28}$ INTRODUCTION}

${ }_{29}$ The selective interaction and pair formation of nucleic acid so polymers and oligomers is the basic mechanism enabling ${ }_{31}$ gene coding and replication. It also at the core of a wealth ${ }_{32}$ of other biological processes, such as gene regulation and ${ }_{33}$ secondary structuring of RNA, of biotechnologies, such as ${ }_{34}$ PCR and SELEX, and of DNA-based nanotechnologies and ${ }_{35}$ DNA origami. These interactions are based, for the largest ${ }_{36}$ part, on the Watson-Crick pairing of complementary bases ${ }_{37}$ (1). DNA and RNA pairing has a limited interval of stability. ${ }_{38}$ When submitted to enough stress, either physical (tempera39 ture, competing forces) or chemical (solvent composition) the 40 double helix denaturates. In particular, thermal denaturation, ${ }_{41}$ leading to the unfolding of a single strand or to the splitting of 42 a duplex, is of paramount importance in technologies, includ${ }_{43}$ ing amplification, screening and sequencing (2-6). Therein, ${ }_{44}$ tailored nucleic acids sequences usually work as probes un-
${ }_{45}$ veiling biological information of a sample or mediating for 46 amplification during polymerase chain reaction.

$47 \quad$ Thermal denaturation of duplexes provides an easy but ${ }_{48}$ crucial access into DNA and RNA thermodynamics. Melting ${ }_{49}$ curves, typically represented as plots of the fraction of paired ${ }_{50}$ DNA oligomers vs. temperature (T), can be experimentally ${ }_{51}$ accessed either via UV absorbance, based on the so-called ${ }_{52}$ hypochromicity effect (7-11), or by measuring the fluorescent ${ }_{53}$ emission of environment-sensitive DNA or RNA-binding ${ }_{54}$ fluorophores. In both cases, measurements directly yield, after ${ }_{55}$ suitable normalization, the melting curve. Melting curves are ${ }_{56}$ mainly characterized by their characteristic temperature, the ${ }_{57}$ so-called melting temperature $T_{m}$, and by the sharpness of the ${ }_{58}$ bound-unbound transition, both depending on the energetics 59 of pair interactions. While some very simple physical models, 60 based for instance on the Ising model (12) or Haminoltonian ${ }_{61}$ mechanics (13), have been used to describe such interactions, 
${ }_{62}$ the most accepted and used model describing the interaction ${ }_{63}$ of nucleic acid duplexes is the nearest-neighbor (NN) model. ${ }_{64}$ Originally introduced by Tinoco et al. in order to study the ${ }_{65}$ thermostability of RNA $(14,15)$, it has developed into a ${ }_{66}$ detailed protocol enabling to predict $T_{m}$ of strands of arbitrary ${ }_{67}$ length ans sequence. The model has been recently applied ${ }_{68}$ even under circumstances of molecular crowding (16).

69 According to the NN model, the free energy involved in 70 the pairing of two strands depends on the specific nucleotide ${ }_{71}$ sequence and can be obtained as a sum of contributions 72 stemming not just from individual base pairs, but from couples ${ }_{73}$ of nearest base pairs. Such a splitting incorporates the notion 74 that the duplex stability is largely depending on stacking 75 forces, acting between neighbouring molecular planes of ${ }_{76}$ paired bases. The computation of melting temperature $T_{m}$ in 77 the NN model requires knowing the specific contributions to 78 the free energy conveyed by each couple of nearest neighbour 79 paired bases, which depends on the identity and location of so the four nucleobases involved in such a segment of double ${ }_{81}$ helical structure. Conversely, the knowledge of the $T_{m}$ of a 82 wide number duplexes of variable length and sequence enables ${ }_{83}$ determine of the thermodynamic parameters associated with ${ }_{84}$ each type of couples of nearest base pairs (17-26).

The extraction of such thermodynamic parameters nec${ }_{86}$ essarily involves a comparison between experimental and ${ }_{87}$ theoretical melting curves. The latter is typically based on s8 a two-state transition model, which neglects any state in89 termediate between the intact duplex and the fully melted 9o strands (27-29). The theoretical derivation of the melting ${ }_{91}$ curve stands on the calculation of either the system parti${ }_{92}$ tion function or of the equilibrium constant in the balance ${ }_{93}$ equation of the two-state model. The functional shape of ${ }_{94}$ the melting curve slightly varies when considering comple${ }_{95}$ mentary or self-complementary sequences. However, in the 96 latter case, in order to have an agreement between theory and ${ }_{97}$ experiment, the introduction of an extra symmetric correction $98(19,21,22,25,26,28)$ is required.

99 In spite of the success when reproducing experimental data, 100 herein we submit to revision the current formulation of the ${ }_{101}$ theory of DNA melting curves. Specifically, our aim is twofold. ${ }_{102}$ On the one hand, we want to exploit the parallelism between ${ }_{103}$ the problem of DNA melting and the process of disassociation ${ }_{104}$ and recombination of diatomic molecules. From the physical ${ }_{105}$ perspective, they are equivalent problems and the latter has 106 been very well studied in the past $(30,31)$. Specifically, ${ }_{107}$ solutions for the problem involving diatomic molecules have ${ }_{108}$ been obtained with exact mathematical treatment even for ${ }_{109}$ systems with a low number of components, away from the 110 thermodynamic limit. On the other hand, we want to shed 111 some light on the origin of the symmetric correction for 112 self-complementary sequences, which is normally used in ${ }_{113} \mathrm{NN}$ model computations, but lacks of a clean justification. As 114 we will show, in our approach there is no need for such extra 115 entropic contribution.

116

To achieve our goals, we put forward a clear formulation of
117 the melting curve based on the partition function calculation. ${ }_{118}$ We present exact results for arbitrary DNA mixtures that, ${ }_{119}$ afterwards, are thoroughly analyzed in the thermodynamic ${ }_{120}$ limit for systems with experimental relevance.

\section{METHODS}

\section{Partition function for a self-complementary mixture}

${ }_{124}$ We consider a system made by $N$ identical self-complementary ${ }_{125}$ oligomers enclosed in a total volume $V$ at temperature $T$. The ${ }_{126}$ system is characterized thus by a concentration $c=N / V$. ${ }_{127}$ Oligomers can be either free or paired, resulting in a duplex. ${ }_{128}$ Free oligomers give a contribution $G_{f}$ to the global Gibbs free ${ }_{29}$ energy, whereas each duplex contributes with $G_{p}$. Henceforth, so the free energy difference due to the inner degrees of freedom ${ }_{31}$ is $\Delta G_{0}=\Delta H_{0}-T \Delta S_{0}=G_{p}-2 G_{f}$. There is an extra ${ }_{132}$ entropic cost of the pairing, since paired oligomers cannot ${ }_{133}$ explore freely the full phase space. Specifically, we consider ${ }_{134}$ that pairing interaction has a finite range characterized by a ${ }_{135}$ volume $V_{0}$, which formally means that if oligomers $i$ and $j$ ${ }_{136}$ are paired, then the relative position vector $\vec{r}_{i}-\vec{r}_{j}$ of their ${ }_{137}$ centers of mass is enclosed in a volume $V_{0}$.

Since the system is in contact with a thermal bath at temperature $T$, the canonical distribution provides us with the probability $p(n)$ of having $n$ duplexes formed in the system,

$$
\begin{aligned}
p(n) \propto & f(n) \exp \left\{-\beta\left[(N-2 n) G_{f}+n G_{p}\right]\right\} \\
& \times \int_{\mathcal{D}} d \vec{r}_{1} \cdots d \vec{r}_{N} d \vec{p}_{1} \cdots d \vec{p}_{N} \exp \left(-\beta \sum_{i=1}^{N} \frac{\vec{p}_{i} \cdot \vec{p}_{i}}{2 m}\right),
\end{aligned}
$$

where $\beta=\left(k_{B} T\right)^{-1}, k_{B}$ is the Boltzmann constant (which has to be substituted by the gas constant $R$ when the free energy is expressed in molar quantities), $\mathcal{D}$ is the domain of the $6 \mathrm{~N}$ dimensional available phase space, and $f(n)$ is the number of possible different configurations with exactly $n$ duplexes formed in our system (with a total number of oligomers equal to $N$ ). Note that the thermodynamic potential of interest is the Gibbs free energy, which has been previously defined, since we consider that the number of oligomers, the pressure and the temperature is kept constant. Moreover, in this expression, we take into account the degeneracy of the state through $f(n)$, whereas the integral and the first exponential account for the phase space explored by the centers of mass of the oligomers and the inner degrees of freedom, respectively. In the integral of Eq. (1), there are $N-2 n$ free oligomers and $2 n$ paired oligomers. Therefore,

$$
\begin{array}{r}
\int_{\mathcal{D}} d \vec{r}_{1} \cdots d \vec{r}_{N} d \vec{p}_{1} \cdots d \vec{p}_{N} \exp \left(-\beta \sum_{i=1}^{N} \frac{\vec{p}_{i} \cdot \vec{p}_{i}}{2 m}\right) \\
=\left(\frac{2 \pi m}{\beta}\right)^{\frac{3 N}{2}} V^{N}\left(\frac{V_{0}}{V}\right)^{n} .
\end{array}
$$


${ }_{138}$ We thus get that the probability of having $n$ couples of paired 139 oligomers is

$$
p(n)=\frac{1}{Z} f(n)\left[\frac{V_{0}}{V} \exp \left(-\beta \Delta G_{0}\right)\right]^{n},
$$

${ }_{140}$ where we have absorbed conveniently some constant factors ${ }_{141}$ with respect to $n$ in the partition function $Z$. It comes in handy 142 to get rid the appearance of $V_{0}$ from our expressions. To do ${ }_{143}$ that, we write it as an additive entropic contribution, defining 144 the weighted Boltzmann factor

$$
\zeta=[c] \exp (-\beta \Delta G),
$$

${ }_{145}$ where $[c]$ is the number concentration measured in $\mathrm{mol} / \mathrm{L}$ and ${ }_{146} \Delta G=\Delta H-T \Delta S$ is the global free energy, which includes the ${ }_{147}$ effect of $V_{0}$, with $\Delta H=\Delta H_{0}$ and $\Delta S=\Delta S_{0}+k_{B} \ln \left(N_{A}\left[V_{0}\right]\right)$ ${ }_{148}$ with $N_{A}$ being Avogadro's number (the square brackets in $V_{0}$ ${ }_{149}$ just indicates that it is consistently measured in L). Note that, ${ }_{150}$ usually, experimental measurements of the entropy of a duplex 151 hardly distinguishes between the different contributions to the ${ }_{152}$ total entropy change, which makes our choice especially con${ }_{153}$ venient. Importantly, the choice of units for the concentration ${ }_{154}$ and the volume has to be consistent. We have chosen the liter ${ }_{155}$ since this is the unit usually used in literature and consequently ${ }_{156}$ it leads to the values of $\Delta S$ reported in literature. The freedom ${ }_{157}$ to redefine a different factor before the exponential in Eq. 4 158 introducing an extra correcting contributions in $\Delta S$ will play 159 an important role in our final results.

${ }_{160}$ Using the Boltzmann factor introduced above, we obtain ${ }_{161}$ finally that the probability of observing $n$ duplexes, or equiva${ }_{162}$ lently, the probability of being in the hybridization state $n$, is 163

$$
p(n)=\frac{1}{Z} f(n)\left(\frac{\zeta}{N}\right)^{n},
$$

${ }_{164}$ where the partition function guarantees the correct normaliza165 tion, that is,

$$
Z=\sum_{n} f(n)\left(\frac{\zeta}{N}\right)^{n}
$$

${ }_{166}$ The sum above is defined over all possible values of $n$, which ${ }_{167}$ are the integers such that $0 \leq 2 n \leq N$.

${ }_{168}$ At last, we need to write the degeneracy $f(n)$ of an ${ }_{169}$ hybridization state

$$
f(n)=\frac{N !}{2^{n}(N-2 n) ! n !} .
$$

${ }_{170}$ The factors in the denominator takes into account the dif171 ferent symmetries of a hybridization state under exchanging 172 oligomers. Respectively, one can exchange oligomers within 173 each duplex, all the free oligomers, and all the duplexes.

${ }_{174}$ The partition function contains all the relevant statistical 175 information which is useful to derive the expected values for 176 the hybridization state. Specifically one can find straightfor177 ward that the average number of duplexes equals

$$
\langle n\rangle=\zeta \frac{\partial \ln Z}{\partial \zeta} .
$$

178 Later on, we will perform explicit computation of both the 179 partition function and the average number of duplexes. Nev180 ertheless, we focus first on studying a general formalism for 181 analyzing arbitrary mixtures of oligomers.

\section{Partition function for an arbitrary mixture}

${ }_{183}$ In this subsection, we consider the most general system ${ }_{184}$ made by hybridizing oligomers. Specifically, we consider $S$ 185 different sequences labeled from 1 to $S$. We call the number ${ }_{186}$ of oligomers with the sequence $i$ present in our system $N_{i}$. ${ }_{187}$ Therefore, $N=\sum_{i=1}^{S} N_{i}$ is the total number of oligomers 188 in the mixture. Hence, the number concentration of the $i$-th 189 sequence is $c_{i}=N_{i} / V$, whereas again the total concentration ${ }_{190}$ of oligomers is $c=N / V$.

We consider that, in principle, any duplex formation 192 (complementary or not) may occur. In particular, we call ${ }_{193}$ the global free energy change $\Delta G_{i j}$, including the entropic ${ }_{194}$ contribution stemming from the corresponding $V_{0}$, in a duplex 195 formation comprising oligomers with sequences $i$ and $j$. ${ }_{196}$ Consistently, we can define the Boltzmann factors

$$
\zeta_{i j}=[c] \exp \left(-\beta \Delta G_{i j}\right),
$$

197 analogous to Eq. 4. Of course, in a real mixture, some attach198 ments may be very unlikely, which means that such duplex 199 has a very high value of $\Delta G$, or equivalently a very low value 200 of $\zeta$.

In this general case, the hybridization state is not char202 acterized just by one number. On the contrary, we need a ${ }_{203}$ vector $\vec{n}$ containing the number of all formed duplexes $n_{i j}$ ${ }_{204}$ comprising oligomers with sequences $i$ and $j$ in our system. ${ }_{205}$ Note that $n_{i j}=n_{j i}$ by definition. In spite of this dimensional 206 difference, the probability of having a certain hybridization 207 state $p(\vec{n})$ is provided by the canonical distribution

$$
p(\vec{n})=\frac{1}{Z} f(\vec{n}) \prod_{i=1}^{S} \prod_{j=i}^{S}\left(\frac{\zeta_{i j}}{N}\right)^{n_{i j}},
$$

208 with the partition function

$$
Z=\sum_{\vec{n}} f(\vec{n}) \prod_{i=1}^{S} \prod_{j=i}^{S}\left(\frac{\zeta_{i j}}{N}\right)^{n_{i j}} .
$$

${ }_{209}$ On this occasion, the sum over $\vec{n}$ is defined over all possible ${ }_{210}$ values of $n_{i j}$ that are integers and holding the set of inequalities ${ }_{211} 0 \leq 2 n_{i i}+\sum_{j \neq i} n_{i j} \leq N_{i}, \forall i$. Following the same reasoning ${ }_{212}$ that leaded to Eq. 7, the degeneracy $f(\vec{n})$ can be explicitly ${ }_{213}$ written as

$$
f(\vec{n})=\prod_{i=1}^{S} \frac{N_{i} !}{2^{n_{i i}}\left(N_{i}-2 n_{i i}-\sum_{j \neq i} n_{i j}\right) !} \prod_{j=i}^{S} \frac{1}{n_{i j} !} .
$$

214 Again, the partition function contains all the relevant ${ }_{215}$ statistical information from which we can profit to derive 
${ }_{216}$ expected values. In particular, the average number of duplexes 217 comprising oligomers with sequences $i$ and $j$ is given by

$$
\left\langle n_{i j}\right\rangle=\zeta_{i j} \frac{\partial \ln Z}{\partial \zeta_{i j}} .
$$

${ }_{218}$ Note that all the theory converges consistently to the one ${ }_{219}$ developed in the previous subsection for self-complementary 220 mixtures, when considering $S=1$ and with the identification ${ }_{221} n_{11}=n$.

222 We expect that a high level of hetereogenity in the mix${ }_{223}$ ture will lead to greater difficulties in the computation of the ${ }_{224}$ partition function and the successive physical quantities of 225 interest. Remarkably, beside the self-complementary situation 226 with $S=1$, there is another case of particular interest that can ${ }_{227}$ be calculated: when two sequences that are complementary. ${ }_{228}$ In this instance, in fact, the chances of having an energetically 229 convenient hybridization between two identical oligomers ${ }_{230}$ is very unlikely and thus we can consider $\zeta_{11}=\zeta_{22}=0$. ${ }_{231}$ Therefore, the only possible attachment occurs between com${ }_{232}$ plementary oligomers and the hybridization vector becomes ${ }_{233}$ a scalar $n_{12} \equiv n$. In this case, we thus re-obtain the one${ }_{234}$ dimensional theory given by Eq. 6, but with the degeneracy 235 being

$$
f(n)=\frac{N_{1} ! N_{2} !}{\left(N_{1}-n\right) !\left(N_{2}-n\right) ! n !} .
$$

\section{${ }_{236}$ RESULTS}

${ }_{237}$ From now on, we will focus on the two cases introduced ${ }_{238}$ above. Namely, the self-complementary (SC) system and the ${ }_{239}$ complementary couple (CC), neglecting self-hybridization 240 in the latter. Firstly, let us define the melting curve as the ${ }_{241}$ fraction of free oligomers corresponding to the less populated 242 sequence. Therefore, the melting curve is normalized with the ${ }_{243}$ maximum number of duplexes. Taking into account that the 244 maximum number of duplexes for the $\mathrm{SC}$ or $\mathrm{CC}$ cases are $N / 2$ ${ }_{245}$ (we assume an even $N$ for simplicity) or $N_{m}=\min \left(N_{1}, N_{2}\right)$ 246 respectively, we get

$$
M_{c}^{(S C)}=1-\frac{2\langle n\rangle}{N}, \quad M_{c}^{(C C)}=1-\frac{\langle n\rangle}{N_{m}},
$$

247 where we have introduced explicitly in the notation if we are 248 referring to $\mathrm{SC}$ or to $\mathrm{CC}$. Hence, once the system is defined, ${ }_{249}$ that is, the oligomer concentration $c$ and the enthalpic $\Delta H$ 250 and entropic $\Delta S$ changes of duplex formation are known, the ${ }_{251}$ melting curve $M_{c}$ is just a function of the temperature. On the ${ }_{252}$ one hand, for very low temperatures the state with minimum ${ }_{253}$ enthalpy, which corresponds with the maximum number of ${ }_{254}$ duplexes and then $M_{c} \sim 0$, is promoted. On the other hand, 255 entropy is favored for high temperatures and high values of ${ }_{256}$ the melting curve $M_{c} \sim 1$ are expected.

257 In the following, we compute the melting curve for our ${ }_{258}$ two SC and CC mixtures of oligomers. We provide the results 259 both exactly (for finite systems) and in the thermodynamic 260 limit.

\section{Exact results for finite systems}

Herein, we compute exactly the partition function and the melting curve for SC and CC systems. In order to obtain the partition function, we just need to carry out the sum in Eq. 6 with the corresponding degeneracy (either Eq. 7 for the SC case or Eq. 14 for the CC case). Taking into account that the upper bounds of the sum are, respectively $N / 2$ and $N_{m}=$ $\min \left(N_{1}, N_{2}\right)$, and defining conveniently $N_{M}=\max \left(N_{1}, N_{2}\right)$, we get

$$
\begin{aligned}
Z^{(S C)}= & \left(-\frac{2 \zeta}{N}\right)^{\frac{N}{2}} U\left(-\frac{N}{2}, \frac{1}{2},-\frac{N}{2 \zeta}\right), \\
Z^{(C C)}= & \left(-\frac{\zeta}{N_{m}+N_{M}}\right)^{N_{m}} \\
& \times U\left(-N_{m}, 1-N_{m}+N_{M},-\frac{N_{m}+N_{M}}{\zeta}\right),
\end{aligned}
$$

where $U(a, b, x)$ is the confluent hypergeometric function of the second kind also known as Tricomi's function (32). Performing the derivative in Eq. 8 and introducing it in Eq. 15 yields

$$
\begin{aligned}
M_{c}^{(S C)} & =-\frac{N U\left(1-\frac{N}{2}, \frac{3}{2},-\frac{N}{2 \zeta}\right)}{2 \zeta U\left(-\frac{N}{2}, \frac{1}{2},-\frac{N}{2 \zeta}\right)}, \\
M_{c}^{(C C)} & =-\frac{\left(N_{m}+N_{M}\right) U\left(1-N_{m}, 2-N_{m}+N_{M},-\frac{N_{m}+N_{M}}{\zeta}\right)}{\zeta U\left(-N_{m}, 1-N_{m}+N_{M},-\frac{N_{m}+N_{M}}{\zeta}\right)} .
\end{aligned}
$$

262 Remarkably, the expressions above are exact. The prediction 263 should be valid even when the mixture has very few compo264 nent. Of course, real experimental conditions imply $N \gg 1$. 265 Moreover, we expect from the physical point of view that in 266 such a limit, at fixed number concentration $c=N / V, N$ does ${ }_{267}$ not play any significant role as shown in the first three panels 268 of Fig. 1.

\section{${ }_{269}$ Thermodynamic limit}

${ }_{270}$ Since reasonable experimental conditions assure that $N \gg 1$, 271 we focus herein on studying in detail this limit. Furthermore, 272 depending on the arguments, hypergeometric functions are not 273 always easy to compute, which makes even more evident the 274 appropriateness of a more illuminating approach. We consider 275 the thermodynamic limit, which entails the limit $N \rightarrow \infty$ but with the concentration $c=N / V$ remaining fixed.

Since performing asymptotic study of the ratios of hypergeometric functions in Eq. 17 is not an easy task, we approach the limit in an alternative way. Specifically, we use Grassmann variables (33) that allow us to derive the partition function in the thermodynamic limit through saddle point integration (34) after a Hubbard-Stratonovich transformation (35). Doing so, we are able to compute the melting curve in 

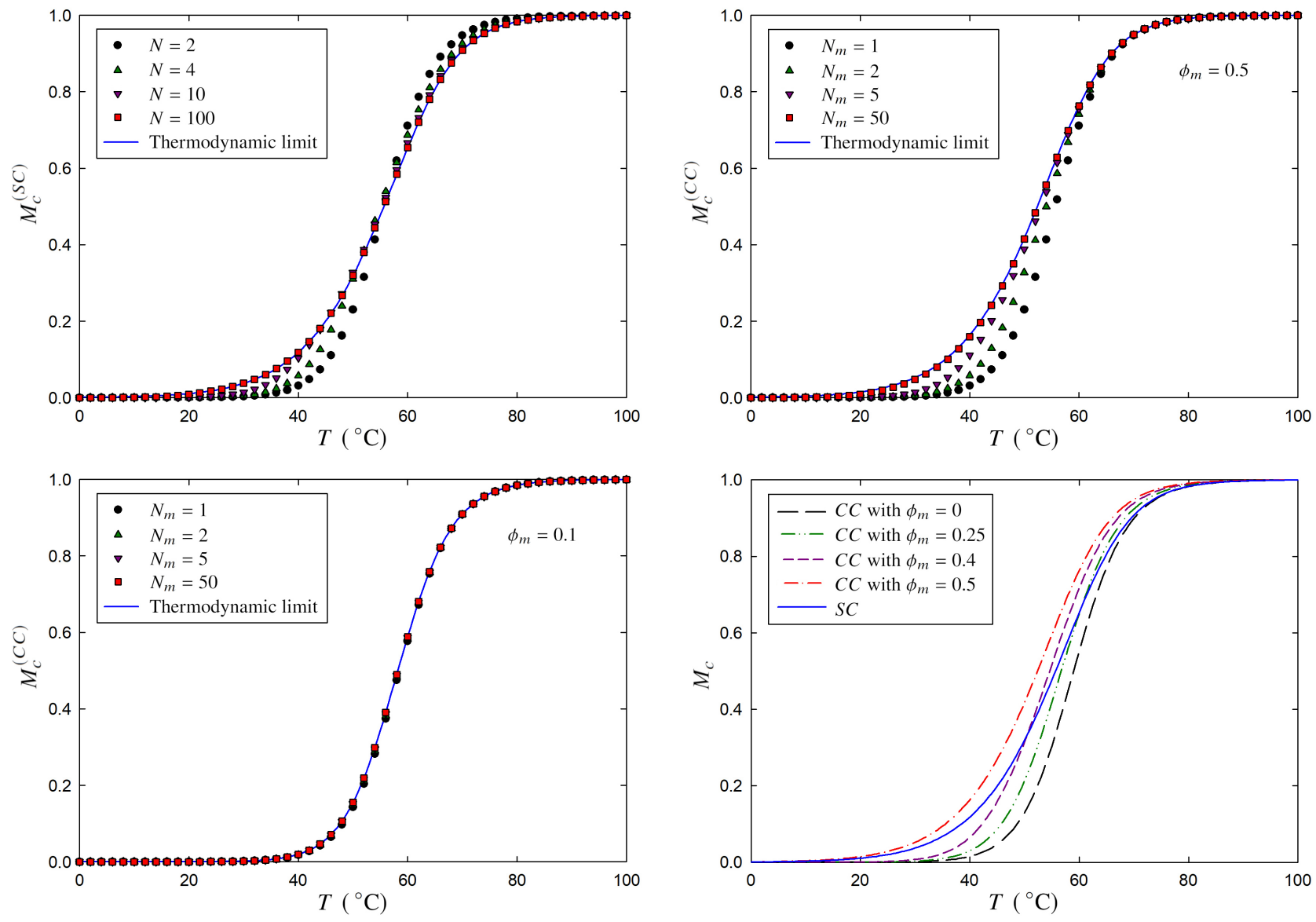

Figure 1: Melting curve for different DNA mixtures. The convergence of the melting curves for finite systems to the predicted thermodynamic limit when the number of constituents is increased is shown in a SC system (top left), a balanced CC system with $\phi_{m}=0.5$ (top right), and an unbalanced CC system with $\phi_{m}=0.1$ (bottom left). Bottom right panel presents a comparison of thermodynamic melting curves for CC systems with different values of $\phi_{m}$ and a SC system. For the numeric evaluation, we have assumed $\Delta H=-45 \mathrm{kcal} \cdot \mathrm{mol}^{-1} \Delta S=-120 \mathrm{cal} \cdot \mathrm{mol}^{-1} \cdot \mathrm{K}^{-1}$ and a concentration of $c=0.4 \mathrm{mmol} / \mathrm{L}$. 
Plata et al.

the thermodynamic limit for both systems of interest, resulting in

$$
\begin{aligned}
M_{c}^{(S C)} & =\frac{2}{1+\sqrt{1+4 \zeta}}, \\
M_{c}^{(C C)} & =\frac{2}{1+\left(1-2 \phi_{m}\right) \zeta+\sqrt{1+2 \zeta+\left(1-2 \phi_{m}\right)^{2} \zeta^{2}}} .
\end{aligned}
$$

${ }_{277}$ where we have introduced the fraction of the less concentrated ${ }_{278}$ sequence $\phi_{m}=N_{m} / N$. The details of the calculations are ${ }_{279}$ provided in the Supporting Material. The convergence of the 280 melting curve to the predicted thermodynamic limit is shown ${ }_{281}$ in the three first panels of Fig. 1

\section{${ }_{282}$ Melting temperature}

In the context of DNA hybridization and melting, the most analyzed quantity is the melting temperature $T_{m}$. The melting temperature is simply defined as the temperature in which half of duplexes are formed. According to our definition of melting curve, the melting temperature is obtained when we equal $M_{c}=0.5$, resulting in

$$
\begin{aligned}
T_{m}^{(S C)} & =\frac{\Delta H}{k_{B} \ln \left(\frac{[c]}{2}\right)+\Delta S}, \\
T_{m}^{(C C)} & =\frac{\Delta H}{k_{B} \ln \left(\frac{2-3 \phi_{m}}{2}[c]\right)+\Delta S} .
\end{aligned}
$$

\section{${ }_{283}$ DISCUSSION}

${ }_{284}$ Now, we highlight the most significant facts that stem from ${ }_{285}$ the results derived in the previous section. In Fig. 1, we have ${ }_{286}$ illustrated several melting curves for both, SC and CC, cases ${ }_{287}$ either in systems with finite size or in the thermodynamic 288 limit. Specifically, we can observe how the convergence to ${ }_{289}$ the thermodynamic limit, when increasing the number of ${ }_{290}$ constituents, is perfect in the first three panels. Remarkably, ${ }_{291}$ as seen in the bottom left panel of 1 , the effect of the finite 292 size almost disappears for very unbalanced $\mathrm{CC}$ systems, that ${ }_{293}$ is, with low values of $\phi_{m}$. This is in fact intuitive since, in 294 this limit, the less concentrated sequence can be considered ${ }_{295}$ as in contact with a bath of strands of the more concentrated ${ }_{296}$ sequence. Therefore, the finite size becomes irrelevant since ${ }_{297}$ the population of the more concentrated sequence takes rel298 atively high values, legitimating the thermodynamic result, 299 even for low values of $N_{m}$. In other words, if we consider a 300 very unbalanced case, that is, $\phi \ll 0.5$, although $N_{m}$ could be 301 low, $N$ will take high values. Finally, in the bottom right panel, 302 we have plotted several melting curves in the thermodynamic 303 limit of CC systems for different fractions of the less concen304 trated sequence $\phi_{m}$, as well as the thermodynamic limit of an 305 equivalent SC system. For all the numerical evaluations of 306 the melting curves, we have assumed $\Delta H=-45 \mathrm{kcal} \cdot \mathrm{mol}^{-1}$,
${ }_{307} \Delta S=-120 \mathrm{cal} \cdot \mathrm{mol}^{-1} \cdot \mathrm{K}^{-1}$ and a total concentration of ${ }_{308} c=0.4 \mathrm{mmol} / \mathrm{L}$. The thermodynamic parameters considered 309 are consistent with the order of magnitude of complemen310 tary sequences around a length of 6 bases at a reference salt 311 concentration of $1 M(26)$.

\section{Exact results for finite systems}

${ }_{313}$ Our exact theory culminating in Eq. 17 provides an analytical 314 prediction for the melting curve in systems with finite size ${ }_{315}$ either SC or CC. Considering equivalent free energies and ${ }_{316}$ concentrations, the $\mathrm{SC}$ system will be more stable than the ${ }_{317}$ balanced CC case. In a SC system, all components are available ${ }_{318}$ for hybridization, whereas the identity of the oligomer in the ${ }_{319}$ CC system is important. Nevertheless, there is a curious ${ }_{320}$ unique situation where both of them converge to the same ${ }_{321}$ phenomenology. This is when we compare a SC system with ${ }^{322} N=2$ and a $C C$ system with $N_{1}=N_{2}=1$. Under this ${ }^{323}$ assumption, the partition function in both cases becomes 324 simply

$$
Z=1+\zeta
$$

325 thus obtaining the melting curve

$$
M_{c}=\frac{1}{1+\zeta} .
$$

${ }_{326}$ Our theory, as it must be, reflects this consistency. In particular, ${ }_{327}$ the melting curves plotted with black circles in the top panels ${ }_{328}$ of Fig. 1 are completely identical.

${ }_{329}$ For the best of our knowledge, it is the first time that 3зо exact results, beyond the thermodynamic limit, are derived ${ }_{331}$ in the context of DNA melting. Nevertheless, as stated in the ${ }_{332}$ Introduction, the problem of DNA hybridization resembles ${ }_{333}$ the (dis)association problem in the field of chemical reactions. ${ }_{334}$ Specifically, the SC and CC case analyzed in this work have a ${ }_{335}$ direct parallelism with the formation of diatomic molecules ${ }_{336}$ either homonuclear or heteronuclear respectively. In spite ${ }_{337}$ of a different approach, based on computing the stationary ${ }_{338}$ solution of the Master Equation describing the dynamics of 339 the reaction, the results derived by McQuarry (30) in the ${ }_{340}$ context of chemical reactions are fully consistent with ours.

\section{${ }_{341}$ Thermodynamic limit}

${ }_{342}$ The convergence of the exact result for increasing values of ${ }_{343} N$ to the derived thermodynamic limit shown in the first three ${ }_{344}$ panels of Fig. 1 represents a direct validation of our asymptotic ${ }_{345}$ calculations. On the one hand, the result for the melting curve 346 in the case of CC systems matches with those provided in ${ }_{347}$ the literature. Specifically, most of the time, one finds the ${ }_{48}$ results derived in the particular case of a balanced CC system, ${ }^{49}$ with $\phi_{m}=0.5$. Our result goes beyond the balance case of ${ }_{50} \mathrm{CC}$ mixtures, explaining the role of the relative concentration ${ }_{51}$ through the fraction $\phi_{m}$. This is especially clear in the bottom ${ }_{352}$ right panel of Fig. 1, where we have displayed the melting ${ }_{353}$ curves for the CC system using different values of $\phi_{m}$ as well as 
354 we compare with the melting curve for the SC system. Therein, 355 we observe, as stated above, that the SC system is more stable ${ }_{356}$ than the balanced CC case, that is, the melting curve for the ${ }_{357}$ first remains below the one for the latter. Furthermore, we ${ }_{358}$ can see how the melting curve for $\phi_{m}=0$ acts as a lower 359 bound for the SC case. Note that when we write $\phi_{m}=0$, ${ }_{360}$ we are considering the limit of $\phi_{m} \rightarrow 0$ after taking the ${ }_{361}$ thermodynamic limit. Indeed, in this extremely unbalanced ${ }_{362}$ case there is a bath of available strands to be paired. On ${ }_{363}$ the other hand our result for the melting curve for the SC ${ }_{364}$ case differs from those traditionally found in the literature 365 (28). Therein, one finds a factor 8 accompanying $\zeta$ in the 366 denominator instead of the factor 4 written in Eq. 18.

367 Such a mismatch is quite curious. On the one hand, our ${ }_{368}$ approach (with the 4) is the correct asymptotic limit of the 369 exact results that are consistent with the theory developed ${ }_{370}$ for chemical reactions. On the other hand, the traditional ${ }_{371}$ melting curve in the literature (with the 8 ) has been used inten372 sively explaining and fitting data from real experiments. This ${ }_{373}$ leads to the question: what is special in self-complementary 374 sequences?

${ }_{375}$ From the physical point of view, a duplex is characterized 376 by its $\Delta G$, which, apart from the temperature, is defined by 377 its enthalpy $\Delta H$ and its entropy $\Delta S$. For both cases, either ${ }_{378} \mathrm{SC}$ or $\mathrm{CC}$, there is just a single way for the coupling of two 379 oligomers, due to the directionality 5' -3 ' of the single strand 380 DNA. Therefore, there is no physical intuition for stating that ${ }_{381}$ the biophysical mechanisms of hybridization are different 382 when the oligomers are self-complementary. However, in the ${ }_{383}$ tables of thermodynamic parameters for $\mathrm{SC}$ and $\mathrm{CC}$ present in 384 literature, an entropic symmetry correction is systematically ${ }_{385}$ applied to self-complementary sequences $(19,21,22,25,26$, 386 28). This correction $\Delta S_{\text {sym }}$ is such that $\exp \left(\Delta S_{\text {sym }} / k_{B}\right) \simeq 0.5$. ${ }_{387}$ In other words, this artificial correction transforms the wrong ${ }_{388}$ factor 8 into the correct one 4.

звя Of course, from the practical point of view, there is no 390 difference between using the traditional melting curve after 391 applying the symmetric correction or using directly our result 392 without adding any artifact. Nevertheless, from our point 393 of view, it is important to highlight that there is neither 394 a real extra entropic contribution nor a special physics in 395 self-complementary DNA.

\section{${ }_{396}$ Reaction equations approach}

${ }_{397}$ The traditional result for the SC case has been usually derived 398 from equilibrium constant arguments. Herein, we give a brief 399 alternative approach which highlight where the mismatch 400 emerges. Specifically, we consider the reaction

$$
2 A \underset{k_{b}}{\stackrel{k_{f}}{\rightleftharpoons}} A_{2},
$$

${ }_{401}$ which represents the (dis)association of homonuclear diatomic 402 molecules, which also describes the hybridization of self${ }_{403}$ complementary systems. We write down the macroscopic
${ }_{404}$ evolution equation, that is, neglecting fluctuations, for the ${ }_{405}$ number of free atoms $N_{a}$,

$$
\frac{d N_{a}}{d t}=-k_{f} \frac{N_{a}\left(N_{a}-1\right)}{V}+k_{b}\left(N-N_{a}\right) .
$$

${ }_{406}$ In the equation above, accompanying the forward rate $k_{f}$, we ${ }_{407}$ find the number of possible couples in the system normalized 408 by the volume which is $N_{a}\left(N_{a}-1\right) / 2 V$ that multiplies the ${ }_{409}$ number of atoms that disappear, that is, 2 . In turn, along 410 with the backward rate $k_{b}$, we have the number of duplexes ${ }_{411}\left(N-N_{a}\right) / 2$ that multiplies the number of appearing atoms, 412 which is again 2.

${ }_{413}$ The equilibrium constant, defined as the ratio of the for${ }_{414}$ ward rate, yields $K=k_{f} / k_{b}=\exp (-\beta \Delta G)$. Introducing this 415 relation, the definition of the melting curve $M_{c}=N_{a} / N$ 416 and the stationary solution of Eq. 23, then the relation ${ }_{417} \zeta=\left(1-M_{c}\right) / M_{c}^{2}$ is obtained, where we have made use ${ }_{418}$ of the definition of $\zeta$ in Eq. 4 and neglected terms of order ${ }_{419} N^{-1}$. Solving this equation for $M_{C}$ leads straightforwardly to ${ }_{420}$ our result for the SC melting curve in Eq. 18. In the traditional ${ }_{421}$ derivation, the mismatch stems from taking blindly the equi422 librium constant as the fraction between the concentration of ${ }_{423}$ duplexes and the squared concentration of the single strands. ${ }_{424}$ Such misconception is equivalent to assume in our equa${ }_{425}$ tions that the forward process is proportional to the product ${ }_{426} 2 N_{a}\left(N_{a}-1\right) / V$ instead of $N_{a}\left(N_{a}-1\right) / V$. This is not correct ${ }_{427}$ since it means that the possible couples of free particle are 428 counted twice.

\section{${ }_{429}$ CONCLUSION}

${ }_{430}$ In this work, we have put forward the fundamentals of statis${ }_{431}$ tical physics to the service of the problem of DNA melting. ${ }_{432}$ We have provided an equilibrium description of hybridization ${ }_{433}$ and melting for nucleic acids, deriving the partition function ${ }_{434}$ in any arbitrary mixture made by DNA.

We have then focused on two experimentally relevant ${ }_{436}$ systems, made by either self-complementary sequences (SC) ${ }_{437}$ or different complementary couple (CC) of strands. In such ${ }_{438}$ cases, the exact melting curves have been derived and we have 439 shown that our results agree with the known expressions in ${ }_{440}$ the context of chemical reactions. Remarkably, this implies ${ }_{441}$ that there is a factor two mismatch with the literature of DNA ${ }_{442}$ melting. Such a discrepancy has led us to debunk the artificial 443 entropic correction applied to self-complementary duplexes 444 in the literature, which we have now clearly identified as an ${ }_{445}$ artifact performed in order to reconcile experimental data 446 with the theory.

${ }_{447}$ Beyond our exact result, we have derived the melting curve 448 of the systems of interest in the thermodynamic limit, which ${ }_{449}$ is the meaningful situation in real experimental conditions. ${ }_{450}$ As expected, the convergence to the thermodynamic limit ${ }_{451}$ when considering the exact result with increasing number of 452 constituents is excellent.

Although the computation of the melting curve for the SC 
${ }_{454}$ and CC cases has been made by considering only complemen- 500 ${ }_{455}$ tary sequences (and thus just one single way for the attachment ${ }_{501}$ ${ }_{456}$ between pairs of DNA strands), our model can be generalized ${ }_{502}$ ${ }_{457}$ to describe pairs with a preferential ways of attachment, even ${ }_{503}$ ${ }_{458}$ if not complementary. The lack of full complementarity would ${ }_{504}$ 459 lead to lower values of $\zeta$, resulting in a system with lower ${ }_{460}$ stability and a melting curve shifted to lower temperatures. ${ }_{461}$ The exact calculations for such more heterogeneous cases is ${ }^{50}$ ${ }_{462}$ left for future investigations.

\section{${ }_{463}$ AUTHOR CONTRIBUTIONS}

${ }_{464}$ S.S. and T.B. designed the research. A.M. provided the original ${ }_{465}$ idea for the analytical approach. C.A.P. and S.M. performed ${ }_{466}$ the research. All authors contributed to the manuscript writing.

\section{${ }_{467}$ ACKNOWLEDGMENTS}

${ }_{468}$ S.S. and C.A.P acknowledge UNIPD STARS grant BioReact ${ }_{469}$ 2018. T.B. acknowledges support by PRIN2017 project from 470 Ministero Istruzione, Università e Ricerca [ID 2017Z55KCW]. ${ }_{471}$ A. Maritan acknowledges the support from University of ${ }_{472}$ Padova through "Excellence Project 2018" of the Cariparo 473 foundation

\section{REFERENCES}

1. Watson, J., and F. Crick, 1953. Molecular Structure of Nucleic Acids: A Structure for Deoxyribose Nucleic Acid. Nature 171:737-738. https://doi.org/10. 1038/171737a0.

2. Ririe, K. M., R. P. Rasmussen, and C. T. Wit- ${ }_{522}^{527}$ twer, 1997. Product Differentiation by Analysis ${ }_{520}$ of DNA Melting Curves during the Polymerase Chain Reaction. Analytical Biochemistry 245:154 - 53 160. http://www.sciencedirect.com/science/ 531 article/pii/S0003269796999169.

3. Reed, G. H., J. O. Kent, and C. T. Wittwer, 2007. High-resolution DNA melting analysis for simple and ${ }^{54}$ efficient molecular diagnostics. Pharmacogenomics ${ }^{53}$ 8:597-608. https://doi.org/10.2217/14622416. ${ }^{536}$ 8.6.597, pMID: 17559349.

4. Cuesta-Lopez, S., D. Angelov, and M. Peyrard, 2009. Adding a new dimension to DNA melting curves. EPL (Europhysics Letters) 87:48009. https://doi . org/10. 1209\%2F0295-5075\%2F87\%2F48009. and A. M. Kaufmann, 2011. The use of melting curves as a novel approach for validation of ${ }_{545}^{544}$ real-time PCR instruments. BioTechniques 51:179184. https://www future-science.com/doi/abs/ 10.2144/000113735, pMID: 21906039. 547
5. Von Keyserling, H., T. Bergmann, M. Wiesel, ${ }^{543}$
6. Balog, J. A., L. Z. Fehér, and L. G. Puskás, 2017. Decoding DNA labels by melting curve analysis using real-time PCR. BioTechniques 63:261266. https://www. future-science.com/doi/ abs/10.2144/000114618, pMID: 29235972.

7. Mandel, M., and J. Marmur, 1968. [109] Use of ultraviolet absorbance-temperature profile for determining the guanine plus cytosine content of DNA. In Nucleic Acids, Part B, Academic Press, volume 12 of Methods in Enzymology, 195 - 206. http: //www. sciencedirect. com/science/article/pii/0076687967121332.

8. Wells, R., J. Larson, R. Grant, B. Shortle, and C. Cantor, 1970. Physicochemical studies on polydeoxyribonucleotides containing defined repeating nucleotide sequences. Journal of Molecular Biology 54:465 497. http://www. sciencedirect.com/science/ article/pii/002228367090121X.

9. Wartell, R. M., and A. S. Benight, 1985. Thermal denaturation of DNA molecules: A comparison of theory with experiment. Physics Reports 126:67 - 107. http://www. sciencedirect.com/science/ article/pii/0370157385900602.

222 10. Mergny, J.-L., and L. Lacroix, 2003. Analysis of Thermal Melting Curves. Oligonucleotides 13:515-537. https: //doi.org/10.1089/154545703322860825, pMID: 15025917.

526 11. Owczarzy, R., 2005. Melting temperatures of nucleic acids: Discrepancies in analysis. Biophysical Chemistry 117:207 - 215. http://www.sciencedirect.com/ science/article/pii/S0301462205001201.

12. Azbel, M. Y., 1979. Phase transitions in DNA. Phys. Rev. A 20:1671-1684. https: //link. aps.org/doi/ 10.1103/PhysRevA.20.1671.

13. Dauxois, T., M. Peyrard, and A. R. Bishop, 1993. Dynamics and thermodynamics of a nonlinear model for DNA denaturation. Phys. Rev. E 47:684-695. https: //link. aps.org/doi/10.1103/PhysRevE.47.684.

${ }_{537}$ 14. Tinoco, I., O. C. Uhlenbeck, and M. D. Levine, 1971. Estimation of Secondary Structure in Ribonucleic Acids. Nature 230:362-367. https://doi.org/10.1038/ $230362 \mathrm{a}$.

54 15. Borer, P. N., B. Dengler, I. Tinoco, and O. C. Uhlenbeck, 1974. Stability of ribonucleic acid doublestranded helices. Journal of Molecular Biology 86:843 - 853. http://www. sciencedirect.com/science/ article/pii/002228367490357X.

546 16. Ghosh, S., S. Takahashi, T. Endoh, H. Tateishi-Karimata,
S. Hazra, and N. Sugimoto, 2019. Validation of 
the nearest-neighbor model for Watson-Crick self- 597 26. SantaLucia, J., and D. Hicks, 2004. The Thercomplementary DNA duplexes in molecular crowd- 598 ing condition. Nucleic Acids Research 47:3284-3294. 599 https://doi.org/10.1093/nar/gkz071.

17. Marky, L. A., and K. J. Breslauer, 1982. Calorimetric determination of base-stacking enthalpies in 6022. double-helical DNA molecules. Biopolymers 21:2185- 603 2194. https://onlinelibrary.wiley.com/doi/ 604 abs/10.1002/bip. 360211107.

18. Petersheim, M., and D. H. Turner, 1983. Base-stacking and base-pairing contributions to helix stability: ther- 60728 . modynamics of double-helix formation with CCGG, ${ }_{608}$ CCGGp, CCGGAp, ACCGGp, CCGGUp, and ACCG- 609 GUp. Biochemistry 22:256-263. https://doi.org/ 610 10.1021/bi00271a004, pMID: 6824629.

19. Breslauer, K. J., R. Frank, H. Blöcker, and L. A. Marky, 612 1986. Predicting DNA duplex stability from the base se- 613 quence. Proceedings of the National Academy of Sciences 614 83:3746-3750. https://www . pnas.org/content/ 615 $83 / 11 / 3746$.

20. Marky, L. A., and K. J. Breslauer, 1987. Calculating 61 thermodynamic data for transitions of any molecularity from equilibrium melting curves. Biopolymers 26:16011620. https://onlinelibrary.wiley.com/doi/ ${ }^{619}$ abs/10.1002/bip. 360260911.

21. Serra, M. J., and D. H. Turner, 1995. [11] Pre- ${ }_{622}$ dicting thermodynamic properties of RNA. In Energetics of Biological Macromolecules, Academic Press, volume 259 of Methods in Enzymology, $242{ }^{624}$ - 261. http://wWw. sciencedirect.com/science/ ${ }^{625}$ article/pii/0076687995590471.

22. Sugimoto, N., S.-i. Nakano, M. Yoneyama, and K.-i. Honda, 1996. Improved Thermodynamic Parameters and Helix Initiation Factor to Predict Stability of DNA Duplexes. Nucleic Acids Research 24:4501-4505. https: ${ }^{60}$ //doi.org/10.1093/nar/24.22.4501. ies from data on nucleic acid oligomers. I. Simple sets of independent sequences and the influence of absent nearest neighbors. Biopolymers 42:783-793.

24. Allawi, H. T., and J. SantaLucia, 1997. Thermodynamics and NMR of Internal G.T Mismatches in DNA. Biochem- ${ }^{637}$ istry 36:10581-10594. https://doi .org/10.1021/ bi962590c, pMID: 9265640.

25. SantaLucia, J., 1998. A unified view of polymer, dumbbell, and oligonucleotide DNA nearest-neighbor thermodynamics. Proceedings of the National Academy of Sciences 95:1460-1465. https://www . pnas . org/ content/95/4/1460.

32. Lebedev, N., and R. Silverman, 1972. Special Functions and Their Applications. Dover Books on Mathematics. Dover Publications. https://books. google.it/ books?id=po-6Yxz851MC.

${ }_{627}$ 33. Berezin, F. A., N. Mugibayashi, and A. Jeffrey, 1966. The Method of Second Quantization. Pure and applied physics : a series of monographs and textbooks. 24. Academic Press. https: //books . google.it/books? $i d=$ fAlRAAAAMAA $J$.

632 34. Bender, C. M., and S. A. Orszag, 1999. Advanced Mathematical Methods for Scientists and Engineers. Springer, New York. modynamics of DNA Structural Motifs. Annual Review of Biophysics and Biomolecular Structure 33:415-440. https://doi.org/10.1146/annurev. biophys.32.110601.141800, pMID: 15139820 .

base-pair changes and cooperativity parameters on the 1425. https://onlinelibrary.wiley.com/doi/ $\mathrm{abs} / 10.1002 / \mathrm{bip} .360220512$.

M. J. Lane, and A. S. Benight, 1997. Predicting sequence-dependent melting stability of short duplex DNA oligomers. Biopolymers 44:217-239. diction of Hybridization and Melting for DoubleStranded Nucleic Acids. Biophysical Journal 87:215 - 226. http://www. sciencedirect.com/science/ article/pii/S0006349504735110.

McQuarrie, D. A., 1967. Stochastic approach to chem
kinetics. Journal of Applied Probability 4:413-478.

. McCoy, B. J., and R. G. Carbonell, 1977. Master equation of diatomic molecules in gases. The Journal of Chemical Physics 66:4564-4571. https://doi.org/10.1063/ 1.433712 .

635 35. Hubbard, J., 1959. Calculation of Partition Functions. Phys. Rev. Lett. 3:77-78. https://link.aps.org/ doi/10.1103/PhysRevLett. 3.77.

\section{SUPPORTING MATERIAL}

${ }_{639}$ An online supplement to this article can be found by visiting ${ }_{640}$ BJ Online at http://www. biophysj . org. 